\title{
Self-management in condition-specific health: a systematic review of the evidence among women diagnosed with endometriosis
}

\author{
Rebecca O'Hara* ${ }^{*}$, Heather Rowe and Jane Fisher
}

\begin{abstract}
Background: Endometriosis is a chronic condition, requiring long-term care as there is no cure. Self-management is the active participation of a person in managing their chronic condition and has been associated with improved knowledge, self-efficacy, performance of self-management tasks and some aspects of health status in interventions for other chronic diseases. The aim was to review the available evidence about the impact of self-management on condition-specific health among women with endometriosis.

Methods: The Medline, PsycINFO, CinahIPlus, Web of Science and Scopus databases were searched and PRISMA guidelines were followed. Search terms were entered both as keywords and mapped to individual database subject headings. Inclusion criteria were: papers that reported investigations of any approach to self-management; among women (at least 18 years) diagnosed with endometriosis and published in English in a peer-reviewed journal. All study designs using quantitative or qualitative methods were eligible for inclusion. Two reviewers independently examined the quality of studies using standard criteria. The systematic review was registered with Prospero (CRD42016042028).
\end{abstract}

Results: A total of 1164 records were identified (after duplicates were removed), and 27 papers, reporting 19 studies met inclusion criteria. Two papers reported findings from RCTs of complementary therapies, seven reported survey data and 18 qualitative studies. No study had investigated all elements of self-management. Women with endometriosis utilise a range of self-care activities and complementary therapies to assist them to manage their symptoms. Women reported both positive and negative experiences with health care providers.

Conclusions: There is some evidence that self-care activities, complementary therapies and positive patienthealthcare provider relationships are important components of self-management for endometriosis. Selfmanagement among women with endometriosis is an emerging field of research and no investigations of all elements of self-management, informed by a comprehensive definition and theoretical framework are available. Health and wellbeing outcomes and barriers and facilitators to self-management for women with endometriosis require further investigation.

Keywords: Self-management, Chronic disease, Endometriosis, women's health, Self-care, Person-centred care

\footnotetext{
* Correspondence: research@beckohara.com.au

School of Public Health and Preventive Medicine, Monash University, Level 4,

553 St Kilda Rd, Melbourne, Victoria 3004, Australia
}

(c) The Author(s). 2019 Open Access This article is distributed under the terms of the Creative Commons Attribution 4.0 International License (http://creativecommons.org/licenses/by/4.0/), which permits unrestricted use, distribution, and reproduction in any medium, provided you give appropriate credit to the original author(s) and the source, provide a link to the Creative Commons license, and indicate if changes were made. The Creative Commons Public Domain Dedication waiver (http://creativecommons.org/publicdomain/zero/1.0/) applies to the data made available in this article, unless otherwise stated. 


\section{Background}

Endometriosis is a chronic, relapsing, inflammatory condition characterised by endometrial-like tissue growing outside the uterus, which can result in adhesions and pain [1-3]. Endometriosis has been associated with pain with menstruation (dysmenorrhea), intercourse (dyspareunia), urination (dysuria), defecation (dyschezia), and ovulation, and lower back and chronic pelvic pain [1, 3]. Other symptoms can include heavy menstrual bleeding, gastrointestinal symptoms, subfertility or infertility, and chronic fatigue $[1,4,5]$. Laparoscopy with histological confirmation is the 'gold standard' for definitive diagnosis of endometriosis [3, 4].

Self-management is key to the effective management of chronic health conditions like asthma, diabetes and arthritis [6]. There is no 'gold standard' definition [6], but self-management is generally conceptualised as the active participation of the person in planning, decision making, and tasks to manage the symptoms, treatment, physical and psychosocial changes involved in living with a chronic condition [6,7]. It extends beyond 'self-care' which is defined as the tasks an individual performs at home in order to manage symptoms of a condition [7]. Key elements of self-management are summarised in Table 1. Barlow and colleagues [6] found that compared to usual care, self-management interventions resulted in improved knowledge, self-efficacy, the performance of selfmanagement tasks and some aspects of health status [6].

Grey and colleagues [11] developed an evidence informed conceptual framework about self- and family management of chronic conditions. It articulates the processes required to undertake self-management including focusing on illness needs (e.g tasks and skills required for the physical management of the condition), activating resources to assist with managing the condition and living with a chronic condition (e.g coping and integrating the condition into life) [11]. Key facilitators and barriers identified in the framework that can affect the ability to self-manage and outcomes include 'personal factors',

Table 1 Key elements of self-management [7-10]

- Active participation in decision making, treatment and management
- Self-care tasks/behaviour change
- Informed decision making
- Psychosocial, emotional or social adjustments
- Monitoring symptoms
- Communication
- Problem-solving
- Patient-provider partnership
- Self-efficacy
- Knowledge of the condition (health literacy)/information seeking
- Resource utilisation

'health status,' 'resources,' 'environment' and the 'healthcare system' [11]. The framework includes 'proximal outcomes' (including 'behaviours,' 'cognitions', 'symptom management' and 'changes in biomarkers') and 'distal outcomes' (including improved 'health status', 'individual outcomes' [e.g. quality of life], 'family outcomes' [e.g. functioning] and 'health care outcomes' [e.g. utilisation]) that result from self- and family management [11].

In relation to endometriosis, previous reviews have focused on women's experiences [12] or the impact of the condition [13]. These have reported some limited findings related to women trialling complementary or self-care activities to manage the disease $[12,13]$. A recent narrative review reported self-management and 'psychological-sexological' interventions in patients with endometriosis [14]. The review concluded that the efficacy of the complementary therapies that were investigated requires further investigation in RCTs and highlighted the importance of a multi-disciplinary team in managing endometriosis. This study did not use standard systematic review methods [15], the search strategy only included the Medline database and references in 'relevant articles', and the search terms were restricted to specific self-care activities and neglected other aspects of self-management, including active decision making, patient-provider partnership, health literacy, and behaviour change.

To date, there has been no systematic review of all the components of self-management in relation to endometriosis. The aim was to describe the evidence about the impact of self-management on condition-specific health among women with endometriosis. The specific objectives were to determine:

a) The aspects of self-management that women with endometriosis undertake to assist them to manage the condition.

b) The association between self-management and health and wellbeing outcomes

c) The barriers to and facilitators of self-management among women with endometriosis.

\section{Methods}

\section{Search strategy}

The review was designed to meet the PRISMA guidelines. A search strategy was developed based on detailed knowledge of the field and in consultation with an expert librarian. The Medline (using the Ovid platform), PsycInfo (using the Ovid platform), CinahlPlus, Web of Science core collection (WOS) and Scopus electronic databases were searched. Search terms were entered both as keywords and mapped to individual database subject headings (where appropriate). Endometriosis was searched using subject headings and keyword term endometrio*. A broad perspective on self-management was adopted to capture 
the multi-faceted nature of this concept and to collate evidence that might not have been labelled 'self-management'. Self-management covers knowledge, behaviours, and activities so over 40 terms were included to capture all elements (see Additional file 1: Appendix A). Reference lists of included papers were manually searched to identify further suitable papers. The systematic review was registered with Prospero (CRD42016042028).

\section{Eligibility criteria}

Inclusion criteria were: reports of investigations of any element of self-management; among women (at least 18 years) diagnosed with endometriosis and published in English in a peer-reviewed journal. All study designs using quantitative or qualitative methods were eligible for inclusion. Exclusion criteria were: investigations of women with pelvic pain or suspected (but not confirmed) endometriosis or data from third parties (e.g health professionals or partners).

\section{Study selection}

A two-stage process for assessing eligibility for inclusion was undertaken. First, an initial search of the literature was undertaken to review the titles and abstracts to identify articles that potentially met the inclusion criteria. Second, the full-text articles were reviewed, and any uncertainties were discussed and agreed upon by consensus among the authors.

\section{Data extraction and quality assessment}

Data elements extracted included: author, year, country of study, research aim, method, recruitment, sample size, sample characteristics, results relating to self-management, barriers or facilitators to self-management and relationship to health and wellbeing outcomes. The quality of papers was assessed using the Standard Quality Assessment Criteria for Evaluating Primary Research Papers (QualSyst) [16]. Both the qualitative and quantitative checklists were used and the highest attainable score for each is 1.0 (Additional file 2: Table S1 \& Additional file 3: Table S2). The quality assessment was completed independently by two authors and consensus was achieved through consultation among them.

\section{Results}

\section{Search results}

The search was conducted on the 13 April 2017 and yielded 2034 records, of which 159 full-text articles were retrieved and assessed for eligibility; 23 met inclusion criteria. A manual search of reference lists from these papers identified a further four records that were included in the review. This yielded a total of 27 papers reporting data from 19 studies (Fig. 1).

\section{Study characteristics}

Of the included papers, two reported findings from RCTs of complementary therapies, seven reported surveys and 18 reported qualitative studies including focus groups or interviews. Most were conducted in high-income countries (US, UK, Sweden, Germany, Netherlands and Australia), but four papers reported findings from Brazil (two RCTs and 1 qualitative study) and South Africa (1 qualitative study), both upper-middle income countries.

Sample sizes for the RCTs were 22 [17] and 40 women [18]. The surveys had samples ranging from 23 to 4000 participants [19-24], one paper did not provide participant summary data [25]. Qualitative studies involved between 13 and 61 women [26-43], and one study also included the partners of women with endometriosis [26].

Reporting of demographic information varied (e.g. average age, age range, descriptive information). Most participants were aged in their 30's. Participants were recruited through gynaecology, hospital, outpatient or GP clinics, or endometriosis support groups or a national endometriosis association. Of the papers that provided sufficient detail, the sample was predominantly educated and partnered. A summary of the study characteristics and the associated quality score is presented in Table 2.

\section{Quality assessment}

Study quality was assessed using QualSyst [16] with the highest possible total score of 1 (Additional file 2: Table S1 \& Additional file 3: Table S2). In addition, evidence of human research ethics committee approval was assessed. Of the 27 papers included, only 19 reported approval from a formally constituted institutional human research ethics committee and seven did not; one paper reported findings from a study that indicated in an associated paper that ethics approval had been obtained.

\section{Quality of RCTs}

Two RCTs investigated complementary approaches to endometriosis pain-management including the use of acupuncture-like and self-applied Transcutaneous Electrical Nerve Stimulation (TENS) [17] and an 8-week yoga program [18]. The quality scores were 0.81 and 0.85. Both used the EHP30 a validated endometriosis specific quality of life measure, and study-specific questions to measure outcomes $[17,18]$. There was too few data for a meta-analysis.

Mira et al. [17] compared two different types of TENS, but it lacked a control group (e.g. sham TENS). The use of the acupuncture-like TENS machine required interaction with a physiotherapist which may have influenced some domains of the EHP30, among women in this group and is a limitation of the study [17]. All participants were retained and assessed at trial endline. 


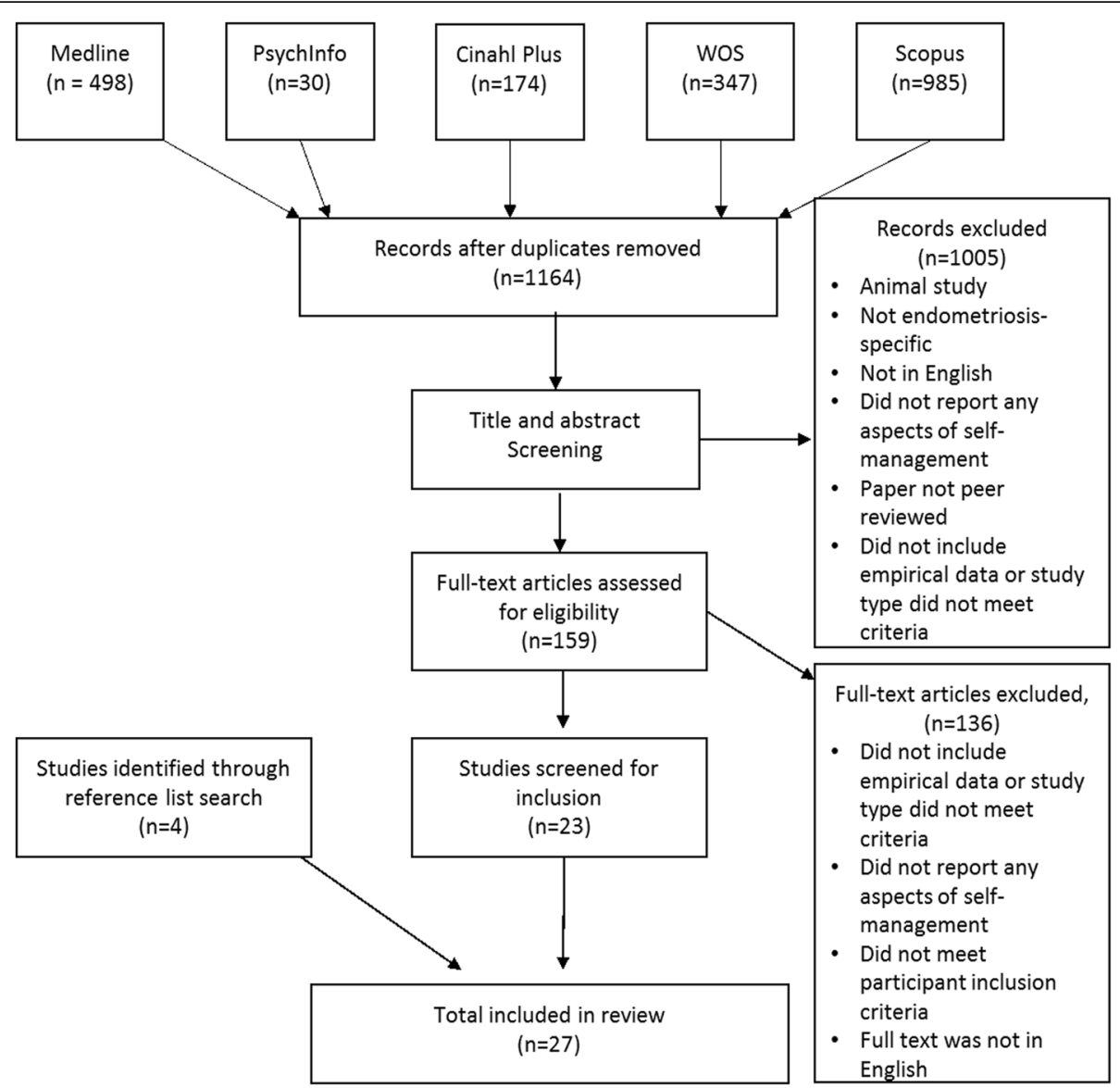

Fig. 1 Systematic review flowchart

Goncalves et al. [18] compared participants of an 8week yoga program to a control condition of no yoga practice. Sample size was calculated from prior research, but there was a high loss to follow up and only $57 \%$ of the yoga group completed the full 8-week program [18].

\section{Quality of survey research}

All the surveys were cross-sectional and quality scores ranged from 0.17 to 0.78 [19-25]. Papers reported descriptive statistics $[19,20,22,25]$ or qualitative analysis (e.g thematic or content analysis) of open-ended survey responses [20, 21, 23, 24]. No studies reported multivariable analyses and one did not report the sample size [25]. Two studies $[19,25]$ did not provide sufficient detail about methods, data collection tools or participants.

\section{Quality of qualitative studies}

The qualitative studies used interviews or focus groups to collect data with quality scores ranging from 0.45 to 0.90 . Only three $[26,31,35]$ considered reflexivity, a core component of qualitative research that acknowledges the role that researchers play as a part of the world they study and its influence on data that are collected [44]. Four studies neglected to report verification procedures (e.g. triangulation, peer review, member checks) associated with analysis that are used to contribute to the rigour of qualitative studies [27, 40-42]. Information about the context of the study, recruitment strategies and question guides was limited, reflecting lower scores on these criteria (Additional file 3: Table S2).

\section{Self-management findings}

A total of nine papers expressly used the term 'self-management' (three papers reporting findings from surveys $[21,23,25]$ and six qualitative papers [36-40, 42]). Formal definitions were not provided with the exception of Roomaney and Kagee [39] who defined self-management as 'steps taken by the participants to alleviate the symptoms of endometriosis'. However, this definition is more consistent with the definition of 'self-care' than that of self-management.

\section{Aspects of self-management}

No study examined all 11 elements of self-management, rather they explored experiences with endometriosis and the needs of women with endometriosis. Findings related to self-management were incidental and were 


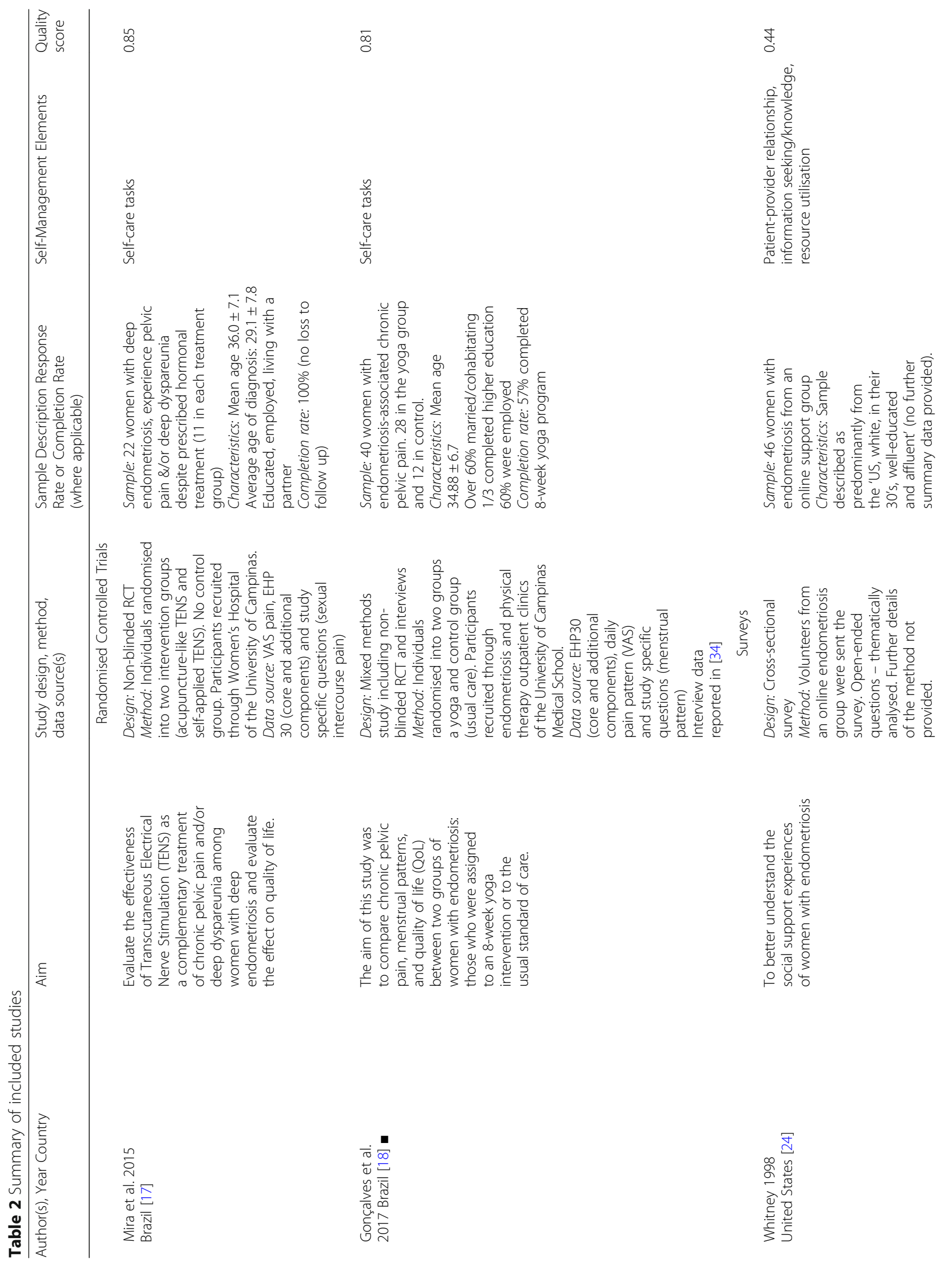




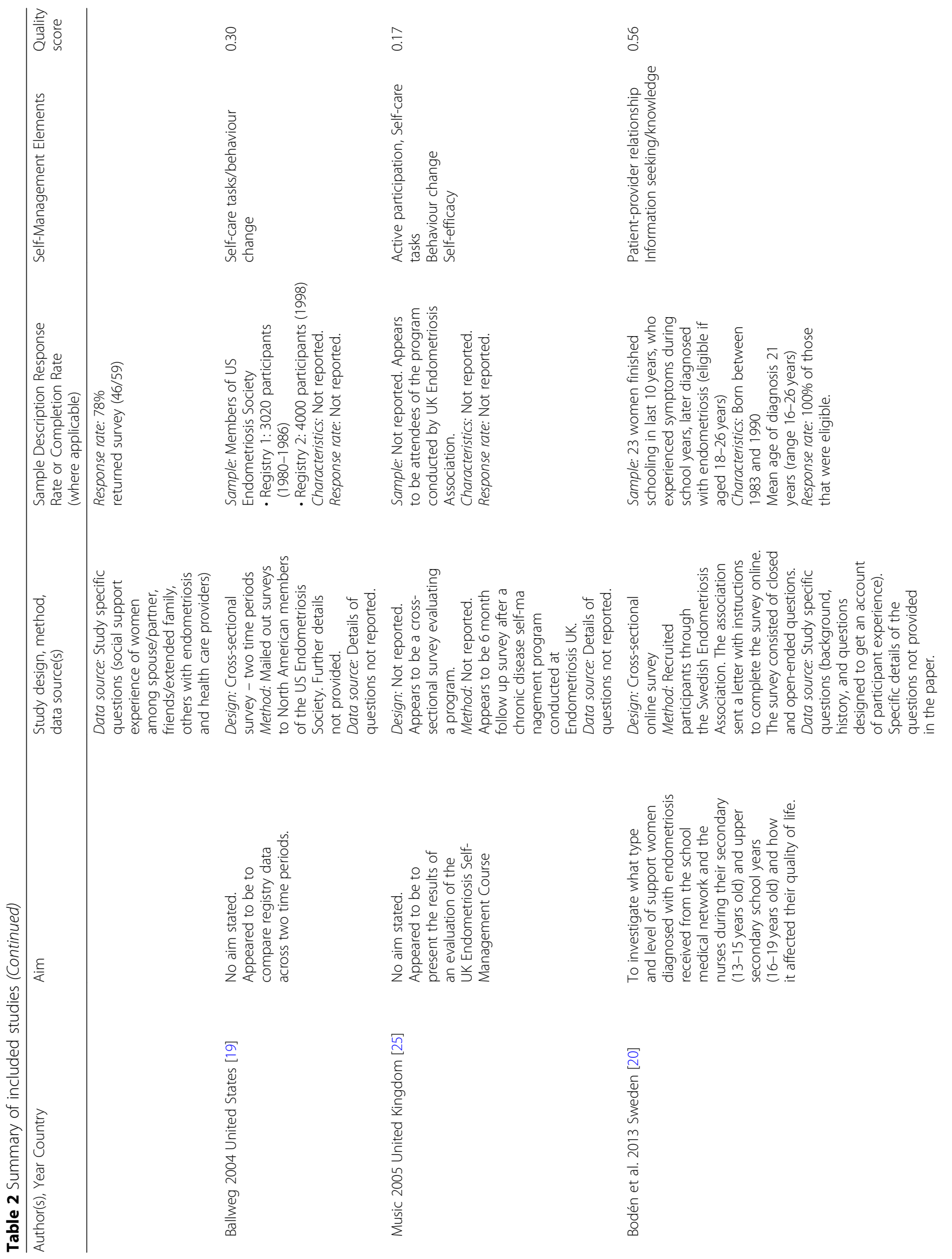




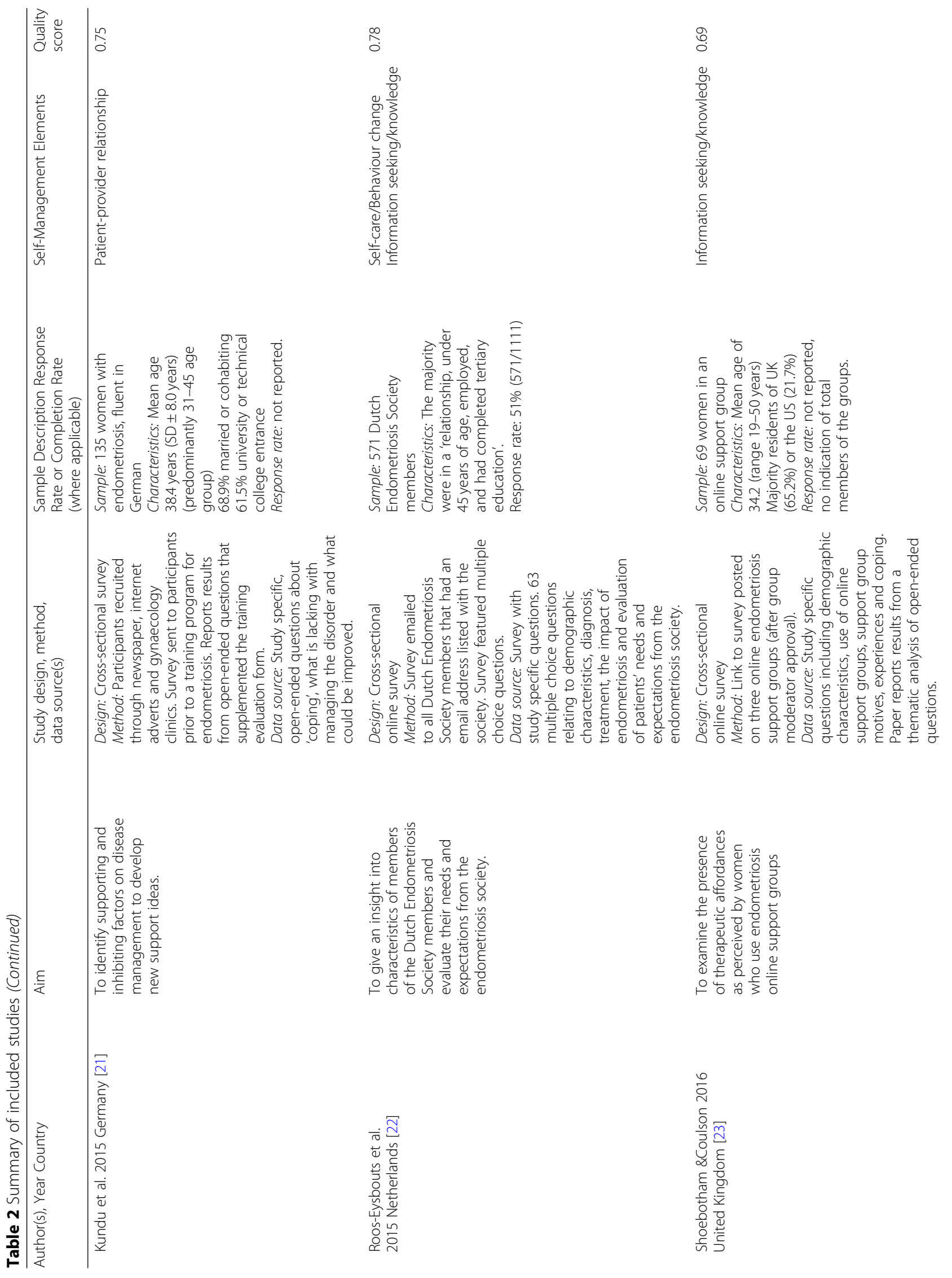




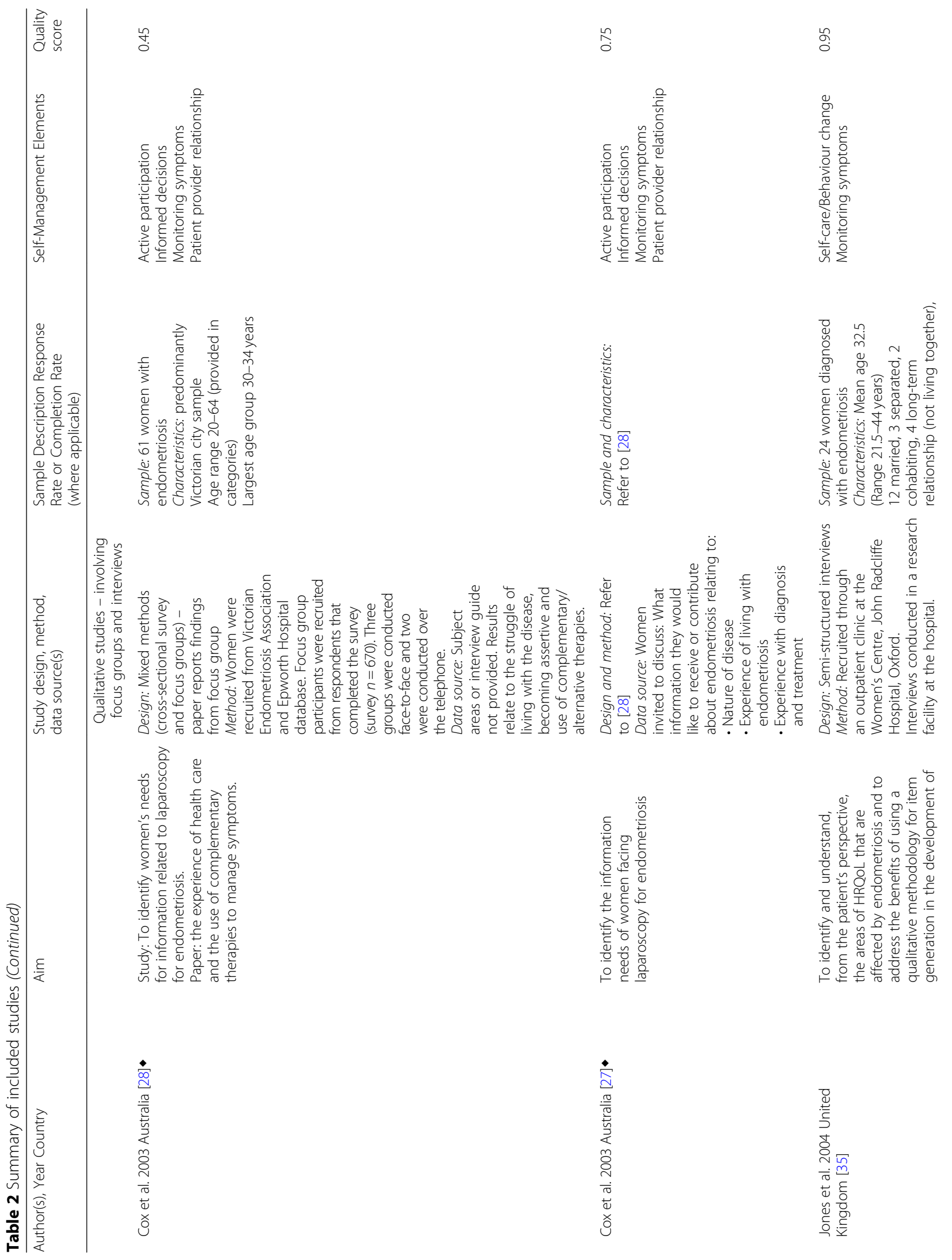




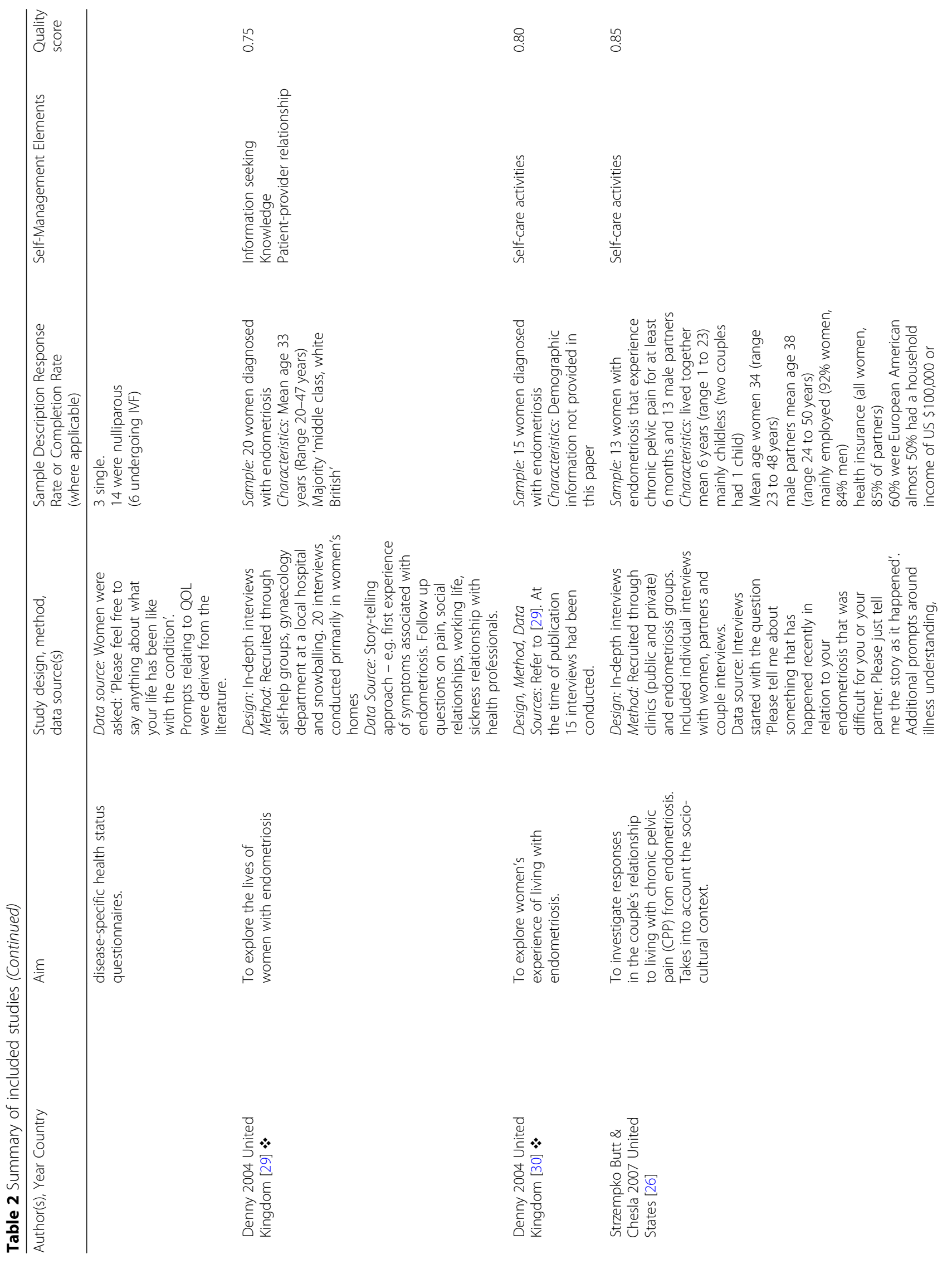




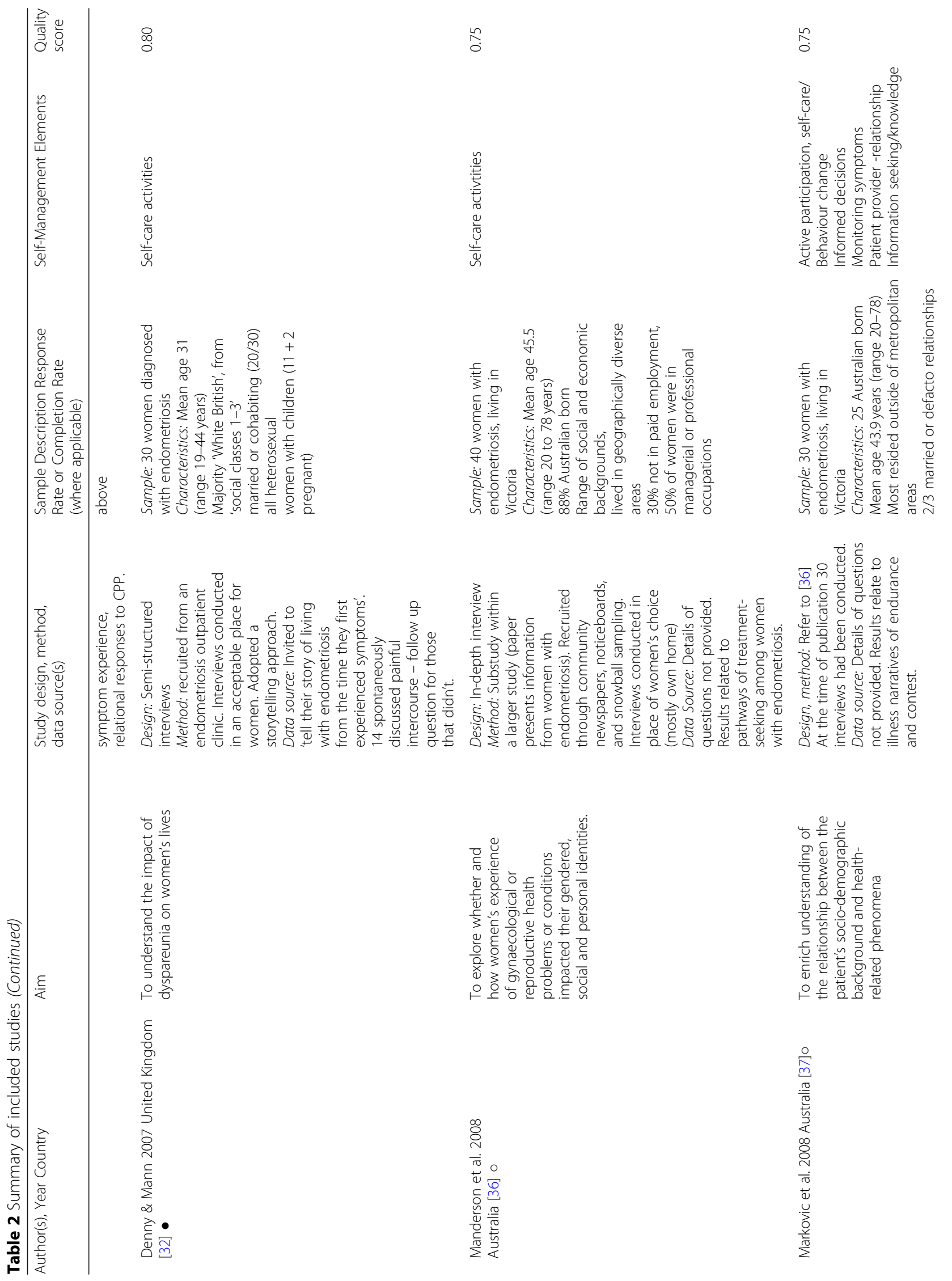




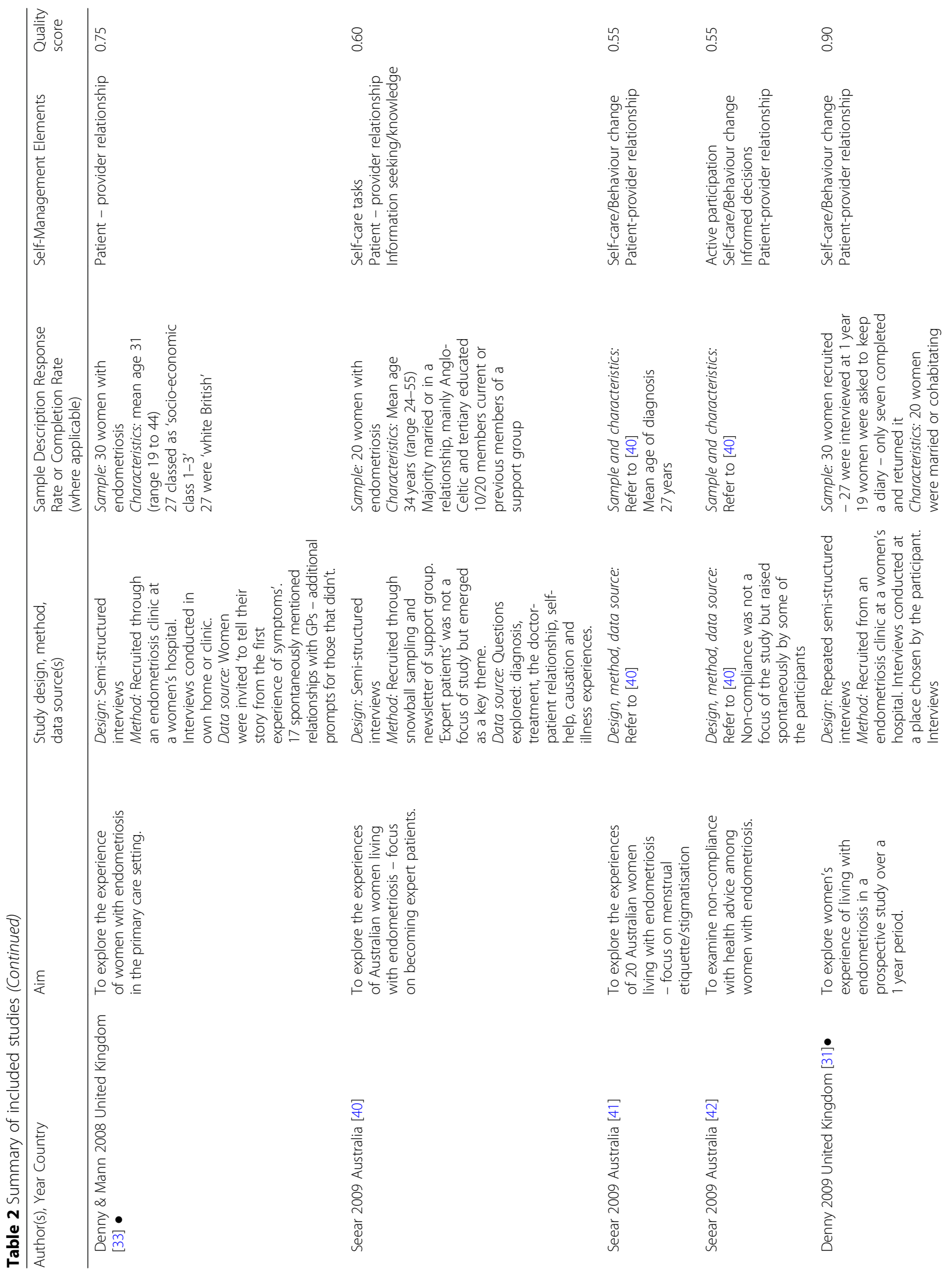




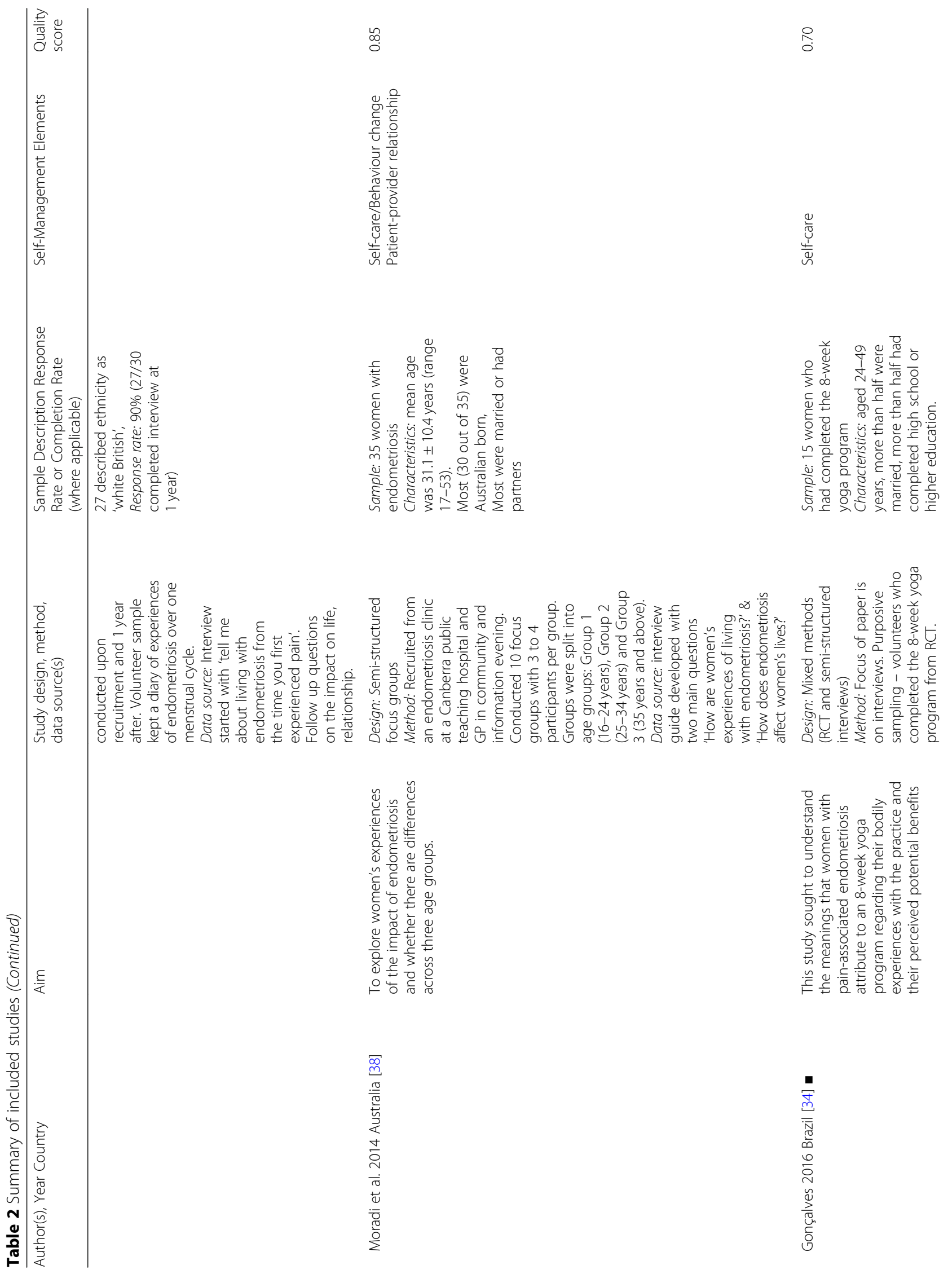




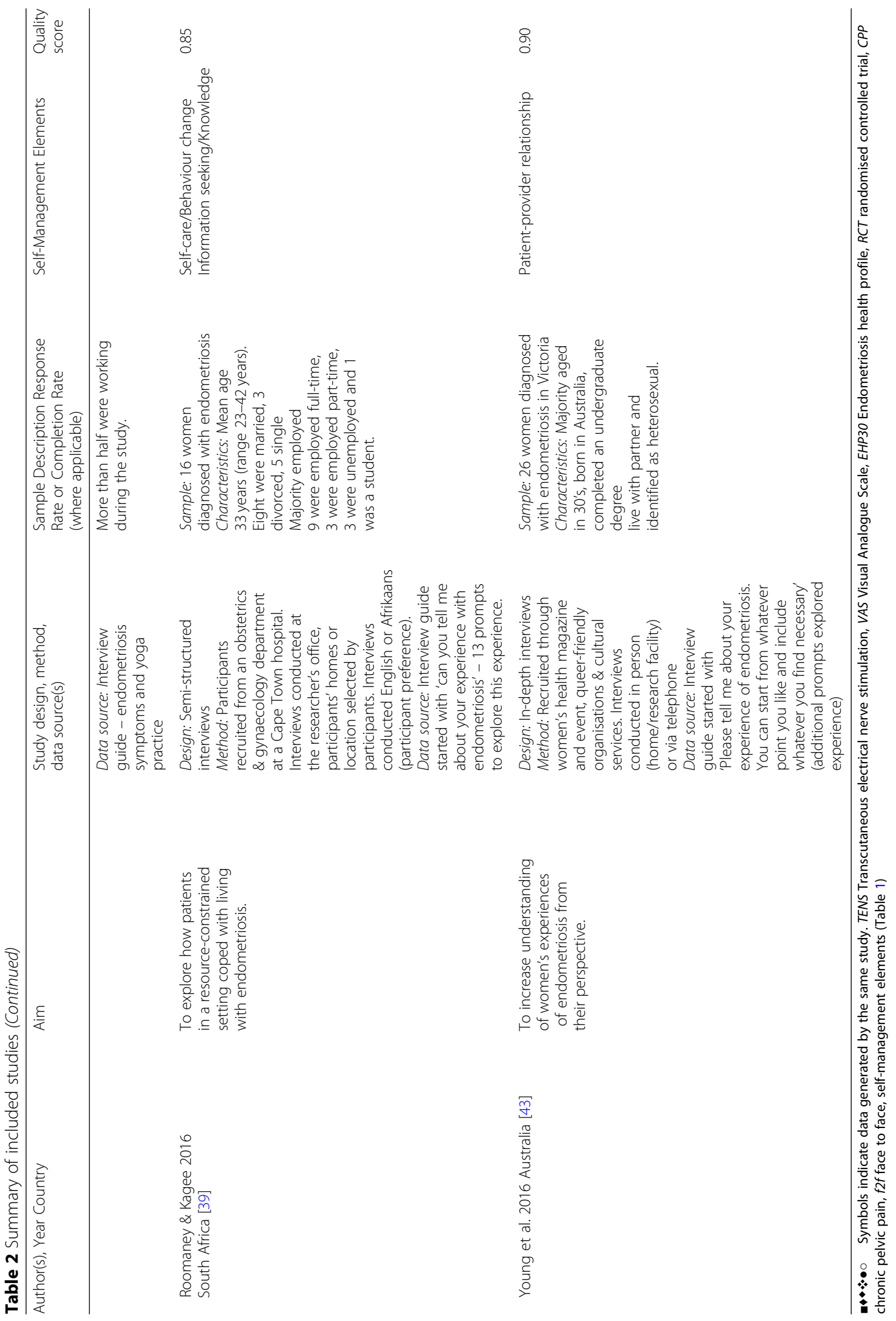


Table 3 Summary of self-care and behaviour change activities

\begin{tabular}{|c|c|}
\hline Activity & Description \\
\hline \multirow[t]{5}{*}{ Lifestyle changes } & - changing diet $[19,26,28,31,38-40]$ \\
\hline & - taking herbs and vitamins $[28,40,42]$ \\
\hline & - exercise $[19,28,38,40]$ (for example yoga $[18,34,40]$, Pilates [19, 42], stretching [39], chi-gong [19]) \\
\hline & - avoiding chemicals (for example making own cleaning products [40], or eating organic food [42]) \\
\hline & • quitting smoking [38]. \\
\hline \multirow[t]{5}{*}{ Cognitive approaches } & • 'positive thinking' $[28,39]$, \\
\hline & - meditation $[26,40]$, \\
\hline & - accepting the disease and learning to live with the condition [39], \\
\hline & • using self-talk to overcome pain [39], \\
\hline & • evoking spirituality (praying or others' prayers) [39] \\
\hline \multirow[t]{7}{*}{ Behaviour change } & $\begin{array}{l}\text { - limiting activity: resting }[26,35,37,39] \text {; not attending social functions }[30,35,38,39] \text {; staying at home } \\
\text { or close to home }[26,38,39] \text {, reducing exercise /sport }[26,38,39] \text {; getting good sleep [38] }\end{array}$ \\
\hline & $\begin{array}{l}\text { - changing work conditions: taking leave }[30,35] \text { or resigning from work [30], working from home [26], } \\
\text { reducing work hours }[42]\end{array}$ \\
\hline & $\begin{array}{l}\text { - sexual activity: avoiding intercourse }[32,34,35,38,41] \text {, adapting sexual position }[26,30,32] \text { or exploring } \\
\text { alternatives to penetrative sex [32], masturbating to orgasm to alleviate pain [36] }\end{array}$ \\
\hline & - scheduling activities for when pain is not as severe $[31,35,39]$ \\
\hline & - reducing stress [40] \\
\hline & • enlisting assistance from others [26] \\
\hline & - managing heavy bleeding: wearing multiple feminine hygiene products [41] \\
\hline \multirow[t]{6}{*}{ Pain management } & • taking analgesics $[22,31,35,36,39,41]$, \\
\hline & • carrying pain medications [26], \\
\hline & • using heat $[31,36,37,39]$, \\
\hline & - massage $[34,36]$ \\
\hline & • TENS machine $[17,31,36]$ \\
\hline & - breathing techniques or relaxation exercises $[34,36,37,39]$ \\
\hline
\end{tabular}

associated with one or more of the elements of selfmanagement (see Table 1).

\section{Relationship with health providers}

Few studies investigated whether a 'partnership' between the woman with endometriosis and the health care provider had been established, rather women's experiences with healthcare providers (e.g. doctors or nurses) were explored [20, 21, 24, 27-31, 33, 35, 37, 38, 41-43].

Positive experiences were associated with the technical competence of the practitioner in endometriosis [21, 24], a 'person-centred' approach [24, 43], pursuit of investigation or treatment options [37, 41], referral to a specialist [33] and effective communication (sharing information and knowledge) [21, 43]. Some women's medical professionals prompted them to take an active role in their healthcare [40], and other women reported that their GP was an 'ally' in the treatment of their disease [29]. Positive experiences with health care providers were associated with a feeling of greater 'control' [24] and a sense of relief as the provider could explain the disease and available options [27].

Four papers reported that women changed providers if they were not satisfied with the care they had received $[27,28,37,43]$. This sometimes required multiple presentations to different health providers [37]. Negative experiences were reported more commonly than positive experiences and were associated with providers lacking knowledge of the condition [20, 27, 29, 33, 35, 42], normalising symptoms as part of menstruation [29-31, 33, $38]$, having poor communication skills [20, 27]; not providing information or providing information not appropriate to women's needs [27, 43], reluctance to refer to specialists [27, 33] and difficulty accessing qualified specialists [38]. Some healthcare providers were described as demonstrating negative attitudes such as doubting women's reports of symptoms, not taking the individual seriously, being dismissive or unsympathetic, or criticising women for not exerting enough effort to manage their endometriosis [20, 27, 29, 31, 33, 37, 38, 42]. When the relationship with the health provider was poor women 
reported they felt neglected [20] or felt they were being dismissed which affected their self-esteem [27].

\section{Information seeking}

Eight papers reported findings in relation to women seeking information about endometriosis, treatments, research, specialists, natural therapies, other management options and surgery [20, 22-24, 29, 37, 39, 40]. Information sources included wide reading, the internet, and support groups or endometriosis associations [20, 22-24, 37]. Being informed about the condition enabled women to 'take charge' and empowered them to make decisions about their health [28]. However, information-seeking also was related to feeling overwhelmed, overloaded, contributed to anxiety about the condition and some women experienced difficulty with conflicting information [40].

Four papers reported findings from women who joined support groups or endometriosis-specific associations $[22-24,40]$. The main reasons for joining these groups was to obtain support, exchange self-help advice (e.g diet and supplements) and the names of appropriate physicians [22-24]. However, Shoebotham \& Coulson [23] concluded that some women had concerns over the quality of information that was shared in these groups. Support groups provided an opportunity to connect with others with the disease, which alleviated feelings of isolation [23, 24, 39]. Positive experiences of groups included improved knowledge, empowerment and quality of life [22, 23]. Providing support to others was beneficial and rewarding [23, 24].

\section{Monitoring symptoms}

Four papers described how some participants recorded and monitored symptoms of their endometriosis with the intention to provide evidence for medical professionals [28, 37], locate patterns (e.g cyclical nature of symptoms) [27], or to predict when symptoms would be severe [35]. Cox et al. [28] reported that recording symptoms provided women with validation that their symptoms where physiological and linked to their menstrual cycle.

\section{Decision making}

Four papers reported findings related to 'taking control' or taking an active role in treatment and management decisions [27, 28, 37, 42]. Cox et al. [27, 28] reported that women were informed about their condition and exercised control over decisions around providers. Two papers reported that women were assertive with health providers, for example by providing proof of the reality of their condition through recorded symptoms [28, 37]. Four papers highlighted that treatment decisions were taken seriously by women, for example about whether to proceed with surgery, or whether to start or continue with medical treatments [27, 28, 37, 42]. Cox et al. [27,
28] discussed that women engaged in goal setting such as being drug-free or achieving good pain management or getting 'off the medical roundabout'. Two papers described women becoming 'experts in their own care' [31, 40]. 'Taking control' of their health and decisions around health enabled women to feel empowered, liberated and allowed them to 'tune in' and listen to their bodies [27, $28,40]$.

\section{Self-care tasks/behaviour change/complementary therapies}

Self-care tasks, complementary therapies and behaviour change were the most widely reported aspects of selfmanagement. Eighteen studies reported accounts of different self-care activities [17-19, 22, 25, 26, 28, 30-32, 3442]. Cox et al. [28] reported that three women of the 61 included in the study had replaced medical management with self-care and alternative therapies. Table 3 outlines the lifestyle changes, cognitive approaches, behaviour change and pain management approaches that women with endometriosis employed.

Lifestyle changes can benefit health [38] but could also result in too much emphasis, lack of enjoyment and changes becoming physically and emotionally demanding $[40,42]$. Seear [40] reported that few women experienced any health benefits following the implementation of these self-care practices. Women reported feeling less confident, or being constantly worried about the condition or resulted in less social contact with other people [30, 35, 38 ], as a result of staying at home or cancelling social events. Avoiding intercourse due to pain resulted in women reporting guilt or inadequacy [35], negative impacts on their relationship [32, 38] and low self-esteem [32].

Seven of the papers reported that women with endometriosis pursued complementary therapies including candidiasis treatment, counselling, acupuncture, homeopathic treatment, naturopathy, lymphatic drainage, massage, Chinese herbal medicine, Reiki, healing touch, aromatherapy, spiritual healing, TENS and Yoga. [17-19, 22, 26-28].

\section{Health and wellbeing outcomes}

Four studies assessed health and wellbeing outcomes (two RCTs investigating the use of TENS and yoga respectively) and two surveys. The other studies did not actively measure health and wellbeing outcomes however, some incidental findings were discussed during the course of investigating women's experiences with endometriosis and the needs of women with endometriosis, which have been summarised in the previous section.

\section{Transcutaneous electrical nerve stimulation}

Both types of TENS machines (acupuncture like and self-applied) provided symptomatic pain relief for chronic pelvic pain and deep dyspareunia [17]. Pain 
with defecation improved with the use of the acupuncture-like TENS [17]. The results from the intervention showed statistically significant improvements in the EHP30 domains for pain, control and powerlessness, emotional wellbeing, social support, self-image, work, sexual intercourse and treatment [17].

\section{Yoga}

There was a statistically significant positive association between yoga and pain relief and quality of life across EHP30 domains of pain, control and powerlessness, emotional wellbeing, self-image, work and treatment. There was no difference between the two groups regarding menstrual patterns. Health and wellbeing outcomes were further elaborated on in the qualitative component of this study [34]. Participants reported that the yoga program was beneficial for controlling pelvic pain through relaxation and breathing techniques [34]. Participants were able to implement breathing and relaxation techniques on their own to respond to pain [34]. Women who completed the yoga program reported an increased level of body awareness and the program also offered social support by connecting with other women with the disease [34].

\section{Self-management program}

One study reported survey findings from an evaluation of a self-management program [25]. The program is a peer-led program involving six weekly sessions, each lasting two and a half hours, covering cognitive pain therapy, dealing with feelings of anger, dealing with fear and frustration, communicating effectively with health care professionals, goal setting, and action planning. After the program participants reported greater confidence, improved quality of life, less anxiety, felt better prepared, greater control in decisions, had reduced the use of pain medications and improved relationships with their health care provider [25].

\section{Self-care/complementary therapies}

Only one survey investigated the efficacy of self-care activities or complementary therapies [19]. The study asked whether a series of specific alternative approaches were 'helpful' but did not elaborate on what was meant by the term 'helpful' [19]. In Ballweg's study [19] 63\% of participants reported that exercise was helpful, $62 \%$ indicated a change in diet, $65 \%$ reported Candidiasis treatment; 59\% counselling; 56\% acupuncture and 56\% indicated vitamin and mineral supplements were 'helpful'.

Facilitators and barriers to self-management

None of the studies specifically investigated facilitators and barriers to self-management. However, some incidental findings were reported in 10 papers $[21,27,28,32,35,37-40,42]$.

\section{Facilitators}

Two facilitators were discussed in the papers which contributed to active engagement in decision making or instigating behaviour change. Four papers reported that experiences with health professionals prompted women to 'take control' of their health, some due to support of providers, whilst others had negative experiences with medical intervention or felt that they had no choice and needed to take action [27, 28, 31, 40]. Women's partners were supportive of behaviour change to avoid pain, for example by avoiding sexual intercourse [32].

\section{Barriers}

Roomaney and Kagee [39] reported that women who lacked knowledge about the disease upon diagnosis needed to learn more about it in order to manage it. A lack of control' and sense of powerlessness was highlighted by Jones et al. [35] as some women reported that they were not able to control the symptoms of endometriosis, which may affect their ability to implement activities to manage the disease. At times women reported that endometriosis symptoms hinder attempts to undertake activities that may be beneficial to their health $[38,42]$ or that it was not always practical to make changes to their lifestyle (e.g. exercise) [42] and that the costs of products or services were prohibitive (e.g. alternative treatment, medicines, diet) $[28,35,40,42]$. Place of residence can be a hindrance to making changes to diet [42] (e.g growing own food) or accessing health professionals [37]. Deficiencies in health professionals' knowledge, empathy, and communication skills were key barriers to the management of endometriosis [21].

\section{Discussion}

Evidence from this review indicates that self-management among women with endometriosis is an emerging field of research. Overall, this body of literature was assessed to be of moderate quality. However, many of the participants in these studies were recruited through a clinic, endometriosis support group or national endometriosis association, which may have resulted in recruitment of participants that were more engaged, or with complex cases or who experience more symptoms compared with women with endometriosis in the general community. Therefore the results should be interpreted with caution as they may have limited generalisability to the wider population of women with endometriosis. Differences in study-specific data collection methods of included studies made comparisons of results difficult. 
The evidence from this review highlights that some aspects of self-management among women with endometriosis such as self-care activities, use of complementary therapies and relationship with providers have been investigated, but the remaining elements of self-management (Table 1) require further investigation. Further, no paper examined all elements of self-management and there were no randomised controlled trials (RCTs) of comprehensive self-management programs compared to usual care among women with endometriosis.

There remains a need for further research into selfmanagement in endometriosis that is informed by a theoretical framework (e.g. the self- and family management framework) [11] and complete definition of selfmanagement. There was limited evidence on health and wellbeing outcomes and facilitators and barriers to selfmanagement, which suggests that these aspects warrant further investigation.

\section{Strengths and limitations of the review}

This review had a number of strengths. First, the search strategy was constructed with an expert librarian and was based on a thorough review of self-management definitions. Second, the systematic review was guided by a published protocol (registered with Prospero CRD42016042028). Third, the quality assessment was directed by a standardised quality assessment tool and two authors independently reviewed the quality of the papers [16]. A limitation of the review is that only studies reported in English were included, and there may be relevant studies in other languages that reported culture-specific information but were missed.

\section{Implications}

\section{Implications for women}

Findings from the review suggest that both complementary therapies [17-19, 22, 26-28] and self-care activities (tasks an individual performs at home in order to manage the symptoms of a condition) [7] are widely used to manage the symptoms of endometriosis. However there was limited evidence as to the efficacy of complementary therapies and self-care activities for managing endometriosis. The findings from two RCTs provide preliminary evidence that Yoga and TENS may be useful for women with endometriosis as both these therapies were associated with significant improvements in physical and psychosocial domains of the EHP30 [17, 18]. Evidence for the utility of self-care practices was mixed Ballweg [19] reported that changing diet, for example, was 'useful' but in Seear's study [40] few women reported experiencing any health benefits following the implementation of selfcare practices. This suggests that women may need to be discerning in the types of therapies they choose and further studies are required to investigate the relationship between additional complementary therapies, self-care activities and improvements in health and wellbeing.

\section{Implications for providers and health care}

Effective patient-provider partnerships are crucial to successful self-management in chronic diseases [7, 9]. Health professionals' knowledge, empathy, and good communication skills assist women to manage their condition but are not always adequate [21] and may prompt a change in provider $[27,28,37,43]$, sometimes multiple times [37]. Whilst no study expressly measured the health and wellbeing impacts of the patient-provider relationship, incidental findings suggest that positive relationships provide women with a 'feeling of control' over the condition [24] and may prompt women to take an active role in their healthcare [29, 40]. Conversely, poor relationships characterised by minimisation of symptoms, or dismissiveness can engender feelings of neglect and abandonment, and damage to self-esteem [20, 27].

There is a need to support providers to integrate practices which promote positive patient-provider partnerships. The findings suggest a need for endometriosisspecific education for providers caring for women with this condition and integration of patient-centred practices. These practices may include providing education to the patient about the condition, identifying problems from the patient's perspective and creating a plan that includes goal-setting and strategies to overcome problems associated with managing the condition [45]. This may necessitate a longer consultation in order to facilitate these discussions, which concurs with Oldroyd et al. [46] findings from GP's that reported longer consultations for chronic disease care are required.

Creating an individualised plan for managing the condition may be a useful mechanism to prompt discussion of specific activities or alternative approaches that women are using to manage the symptoms, given the variety of self-care activities and complementary therapies reported by women with endometriosis in the literature. Providers are encouraged to foster an open discussion, avoid being dismissive or critical for women using these therapies, but encourage women to seek evidence-based therapies.

In a review of chronic disease self-management interventions, Barlow and colleagues [6] found that compared to usual care, self-management interventions are effective in improving knowledge, self-efficacy, the performance of self-management tasks and some aspects of health status [6]. No RCT has been conducted to determine the efficacy of a chronic disease self-management intervention for women with endometriosis. However, Music's evaluation of a self-management program [25] highlighted that after the program participants reported greater confidence, improved quality of life, less anxiety, 
felt better prepared, greater control in decisions, had reduced the use of pain medications and improved relationships with their health care provider. Whilst the findings should be interpreted with caution as this study was of low quality as there was insufficient detail provided about methods, data collection tools or participants, it would suggest that an RCT that compares a self-management intervention to usual care among women with endometriosis may be warranted.

\section{Conclusion}

Self-management is an emerging area of research in endometriosis. The results of this review provide evidence that self-care activities, use of complementary therapies and positive patient-healthcare provider relationships are important components of managing endometriosis. However, further purposeful research is warranted using a clear definition of and comprehensive theoretical framework for self-management. More research is required on health and wellbeing outcomes and facilitators and barriers to self-management among women with endometriosis. An investigation on the efficacy of a selfmanagement program for women with endometriosis is also warranted, given the benefits that have been observed for these programs in other chronic diseases.

\section{Additional files}

\section{Additional file 1: Appendix A. Search strategies for databases.} (DOCX 29 kb)

Additional file 2: Table S1. Quality assessment scores for the papers involving quantitative studies. (DOCX $18 \mathrm{~kb}$ )

Additional file 3: Table S2. Quality assessment scores for the papers involving qualitative methods. (DOCX $20 \mathrm{~kb}$ )

\section{Abbreviations}

GP: General Practitioner; PRISMA guidelines: Preferred reporting items for systematic reviews and meta-analyses; RCTs: Randomised controlled trial; TENS: Transcutaneous Electrical Nerve Stimulation

\section{Acknowledgements}

The authors would like to thank Lorena Romero for her advice and guidance with developing the search strategy for this review.

\section{Authors' contributions}

$\mathrm{RO}$ conducted the search of databases, data extraction, quality assessment and drafting of the paper. JF and HR contributed to the quality assessment and drafting of the paper. All authors have read and approved the final manuscript.

\section{Funding}

$\mathrm{RO}$ is a PhD student and is supported by an Australian Government Research Training Program Scholarship. JF is supported by the Finkel Professorial Fellowship, which is funded by the Finkel Family Foundation. The project was a component of a PhD research program that was supported by the scholarship. The funding bodies had no role in the design of the study, or data collection, analysis, interpretation of data or in writing the manuscript.

\section{Availability of data and materials}

All data generated or analysed during this study are included in this published article [and its supplementary information files].
Ethics approval and consent to participate

Not applicable.

\section{Consent for publication}

Not applicable.

\section{Competing interests}

The authors declare that they have no competing interests.

Received: 6 November 2018 Accepted: 30 May 2019

Published online: 19 June 2019

\section{References}

1. Kennedy S, Bergqvist A, Chapron C, D'Hooghe T, Dunselman G, Greb R, Hummelshoj L, Prentice A, Saridogan E. ESHRE guideline for the diagnosis and treatment of endometriosis. Hum Reprod. 2005;20(10):2698-704.

2. Vermeulen N, De Bie B, Dunselman G. Information for women with endometriosis: patient version of the ESHRE guideline on management of women with endometriosis. Belgium: European Society of Human Reproduction and Embryology; 2014

3. Leyland N, Casper R, Laberge P, Singh S. Endometriosis diagnosis and management: SOGC clinical practice guideline. J Obstet Gynaecol Can. 2010;32(7 (Supplement 2):S1-S32.

4. Dunselman GAJ, Vermeulen N, Becker C, Calhaz-Jorge C, D'Hooghe T, De Bie B, Heikinheimo O, Horne AW, Kiesel L, Nap A, et al. ESHRE guideline: management of women with endometriosis. Hum Reprod. 2014;29(3):400-12.

5. Ek M, Roth B, Ekström P, Valentin L, Bengtsson M, Ohlsson B. Gastrointestinal symptoms among endometriosis patients-a case-cohort study. BMC Womens Health. 2015;15(1):1.

6. Barlow J, Wright C, Sheasby J, Turner A, Hainsworth J. Self-management approaches for people with chronic conditions: a review. Patient Educ Couns. 2002:48(2):177-87.

7. Clark NM, Becker MH, Janz NK, Lorig K, Rakowski W, Anderson L. Selfmanagement of chronic disease by older adults a review and questions for research. J Aging Health. 1991;3(1):3-27.

8. Creer TL, Holroyd KA. Self-management of chronic conditions: the legacy of sir William Osler. Chronic IIIn. 2006;2(1):7-14

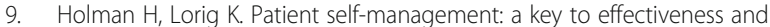
efficiency in care of chronic disease. Public Health Rep. 2004;119(3):239-43.

10. Lorig K, Holman H. Self-management education: history, definition, outcomes, and mechanisms. Ann Behav Med. 2003;26(1):1-7.

11. Grey M, Schulman-Green D, Knafl K, Reynolds NR. A revised self-and family management framework. Nurs Outlook. 2015:63(2):162-70.

12. Young K, Fisher J, Kirkman M. Women's experiences of endometriosis: a systematic review and synthesis of qualitative research. J Fam Plann Reprod Health Care. 2015;41(3):225-34.

13. Culley L, Law C, Hudson N, Denny E, Mitchell H, Baumgarten M, RaineFenning N. The social and psychological impact of endometriosis on women's lives: a critical narrative review. Hum Reprod Update. 2013;19(6): 625-39.

14. Buggio L, Barbara G, Facchin F, Frattaruolo MP, Aimi G, Berlanda N. Selfmanagement and psychological-sexological interventions in patients with endometriosis: strategies, outcomes, and integration into clinical care. Int $J$ Women's Health. 2017:9:281.

15. Moher David, Liberati Alessandro, Tetzlaff Jennifer, Altman Douglas G. Preferred reporting items for systematic reviews and meta-analyses: the PRISMA statement. BMJ. 2009;339:b2535.

16. Kmet LM, Lee RC, Cook LS. Standard quality assessment criteria for evaluating primary research papers froma variety of fields. Alberta: Alberta heritage Foundation for Medical Research; 2004

17. Mira TA, Giraldo PC, Yela DA, Benetti-Pinto CL. Effectiveness of complementary pain treatment for women with deep endometriosis through transcutaneous electrical nerve stimulation (TENS): randomized controlled trial. Eur J Obstet Gynecol Reprod Biol. 2015;194:1-6.

18. Gonçalves AV, Barros NF, Bahamondes $L$. The practice of hatha yoga for the treatment of pain associated with endometriosis. J Altern Complement Med. 2017:23(1):45-52

19. Ballweg ML. Impact of endometriosis on women's health: comparative historical data show that the earlier the onset, the more severe the disease. Best Pract Res Clin Obstet Gynaecol. 2004;18(2):201-18. 
20. Bodén E, Wendel C, Adolfsson A. Adolescents with endometriosis: their experience of the school health care system in Sweden. Br J School Nurs. 2013;8(2):81-7.

21. Kundu S, Wildgrube J, Schippert C, Hillemanns P, Brandes I. Supporting and inhibiting factors when coping with endometriosis from the patients' perspective. Geburtshilfe Frauenheilkd. 2015;75(5):462-9.

22. Roos-Eysbouts Y, De Bie-Rocks B, Van Dijk J, Nap AW. Characteristics, expectations and needs of the dutch endometriosis society members. Gynecol Obstet Investig. 2015;79(4):234-8.

23. Shoebotham A, Coulson NS. Therapeutic affordances of online support group use in women with endometriosis. J Med Internet Res. 2016; 18(5):e109.

24. Whitney ML. Importance of lay organizations for coping with endometriosis. J Reprod Med. 1998;43(3 Suppl):331-4.

25. Music R. Endometriosis and self-management strategies. Womens Health Med. 2005;2(1):38-9.

26. Strzempko Butt F, Chesla C. Relational patterns of couples living with chronic pelvic pain from endometriosis. Qual Health Res. 2007;17(5):571-85.

27. Cox H, Henderson L, Andersen N, Cagliarini G, Ski C. Focus group study of endometriosis: struggle, loss and the medical merry-go-round. Int I Nurs Pract. 2003;9(1):2-9.

28. Cox H, Henderson L, Wood R, Cagliarini G. Learning to take charge: women's experiences of living with endometriosis. Complement Ther Nurs Midwifery. 2003;9(2):62-8

29. Denny E. 'You are one of the unlucky ones': delay in the diagnosis of endometriosis. Divers Health Soc Care. 2004;1 (1):39-44.

30. Denny E. Women's experience of endometriosis. J Adv Nurs. 2004;46(6):641-8.

31. Denny E. I never know from one day to another how I will feel: pain and uncertainty in women with endometriosis. Qual Health Res. 2009;19(7):985-95.

32. Denny E, Mann $\mathrm{CH}$. Endometriosis-associated dyspareunia: the impact on women's lives. J Fam Plann Reprod Health Care. 2007;33(3):189-93.

33. Denny $\mathrm{E}, \mathrm{Mann} \mathrm{CH}$. Endometriosis and the primary care consultation. Eur J Obstet Gynecol Reprod Biol. 2008;139(1):111-5.

34. Gonçalves AV, Makuch MY, Setubal MS, Barros NF, Bahamondes L. A qualitative study on the practice of yoga for women with pain-associated endometriosis. J Altern Complement Med. 2016;22(12):977-82.

35. Jones $\mathrm{G}$, Jenkinson $\mathrm{C}$, Kennedy $\mathrm{S}$. The impact of endometriosis upon quality of life: a qualitative analysis. J Psychosom Obstet Gynaecol. 2004;25(2):123-33.

36. Manderson L, Warren N, Markovic M. Circuit breaking: pathways of treatment seeking for women with endometriosis in Australia. Qual Health Res. 2008;18(4):522-34.

37. Markovic M, Manderson L, Warren N. Endurance and contest: women's narratives of endometriosis. Health (London). 2008;12(3):349-67.

38. Moradi M, Parker M, Sneddon A, Lopez V, Ellwood D. Impact of endometriosis on women's lives: a qualitative study. BMC Womens Health. 2014;14(1):123.

39. Roomaney R, Kagee A. Coping strategies employed by women with endometriosis in a public health-care setting. J Health Psychol. 2016;21(10): 2259-68.

40. Seear K. The third shift: health, work and expertise among women with endometriosis. Health Sociol Rev. 2009;18(2):194-206.

41. Seear K. The etiquette of endometriosis: stigmatisation, menstrual concealment and the diagnostic delay. Soc Sci Med. 2009;69(8):1220-7.

42. Seear K. 'Nobody really knows what it is or how to treat it': why women with endometriosis do not comply with healthcare advice. Health Risk Soc. 2009;11(4):367-85.

43. Young K, Fisher J, Kirkman M. Endometriosis and fertility: women's accounts of healthcare. Hum Reprod. 2016;31(3):554-62.

44. Ahern KJ. Ten tips for reflexive bracketing. Qual Health Res. 1999:9(3):407-11.

45. Coleman MT, Newton KS. Supporting self-management in patients with chronic illness. Am Fam Physician. 2005;72(8):1503-10.

46. Oldroyd J, Proudfoot J, Infante FA, Powell Davies G, Harris MF, Bubner T, Holton C, Beilby JJ. Providing healthcare for people with chronic illness: the views of Australian GPs. Med J Aust. 2003;179:30-3. https://doi.org/10.5694/j. 1326-5377.2003.tb05414.x.

\section{Publisher's Note}

Springer Nature remains neutral with regard to jurisdictional claims in published maps and institutional affiliations.

\section{Ready to submit your research? Choose BMC and benefit from:}

- fast, convenient online submission

- thorough peer review by experienced researchers in your field

- rapid publication on acceptance

- support for research data, including large and complex data types

- gold Open Access which fosters wider collaboration and increased citations

- maximum visibility for your research: over $100 \mathrm{M}$ website views per year

At BMC, research is always in progress.

Learn more biomedcentral.com/submissions 